\title{
The Influence of a Nanometric Layer with a High Refracting Index on the Sensitivity of the Difference Interferometer
}

\author{
K. GuT* \\ Institute of Physics, Silesian University of Technology \\ Krzywoustego 2, 44-100 Gliwice, Poland
}

\begin{abstract}
Planar waveguides in which some range of the waveguide was modified by depositing on it a layer of some other dielectric, are called composite optical waveguides. Such a structure is based on a planar waveguide obtained by means of ion exchange $\mathrm{K}^{+}-\mathrm{Na}^{+}$on glass, and covered by an adequately profiled dielectric layer. This kind of structure is of much interest due to considerable possibilities of taking measurements, which may contribute to a further development of the optoelectronic sensor. Especially required are sensor systems in chemistry, biochemistry, biotechnology, and others. Investigations on the properties of composite layers constitute a continuation of investigations concerning the construction of interferometers, which are very sensitive, cheap and easy to be produced.
\end{abstract}

PACS numbers: 42.82.Et, 07.07.Df, 42.79.Pw

\section{Introduction}

Planar (polarimetric) difference interferometer is a device based on the structure of the planar waveguide [1-3]. The waveguide has been designed as an optic channel where simultaneously two orthogonal modes of the polarization TE and TM are propagated. Due to the difference between the propagation constants of these modes $\Delta \beta=\beta_{\mathrm{TE}}-\beta_{\mathrm{TM}}$, arising from the modal equation, a phase shift $\Delta \varphi$ of both waves is taking place at the output of the waveguide. The interference signal is observed by a polarizer placed at the output of the waveguide, whose vibration plane forms an angle of $45^{\circ}$ with the waveguide surface. The extent of the phase shift $\Delta \varphi_{0}$, which defines the working point of the interferometer, depends on the refractive index of the waveguide cover and on the length of the waveguide structure. Any change in the refractive index of the cover $\mathrm{d} n_{\mathrm{c}}$ will result in a change of the difference of the effective indices $\mathrm{d}(\Delta N)$ and consequently a change

${ }^{*}$ e-mail: kazimierz.gut@polsl.pl 
of the phase shift $\mathrm{d}\left(\Delta \varphi_{0}\right)$, which will in turn result in a change of the interference signal at the output.

In recent years the addition of a new layer was suggested, displaying a larger refracting index on the waveguide structure, in order to increase the sensitivity of the sensors. In order to minimize losses in the transformation of the mode field the thickness of the additional layer is monotonically changed [5-9].

\section{Planar waveguide obtained by means of ion exchange}

The ion exchange involves a modification of the chemical composition of glass as well as a volumetric change of the region near the surface [11]. Stresses arise resulting in birefringence. In order to determine the effective indices of refraction, a different profile of the refraction index must be assumed for the types TE and TM. The profile of the refracting index applied in the calculations has been presented in Fig. 1.
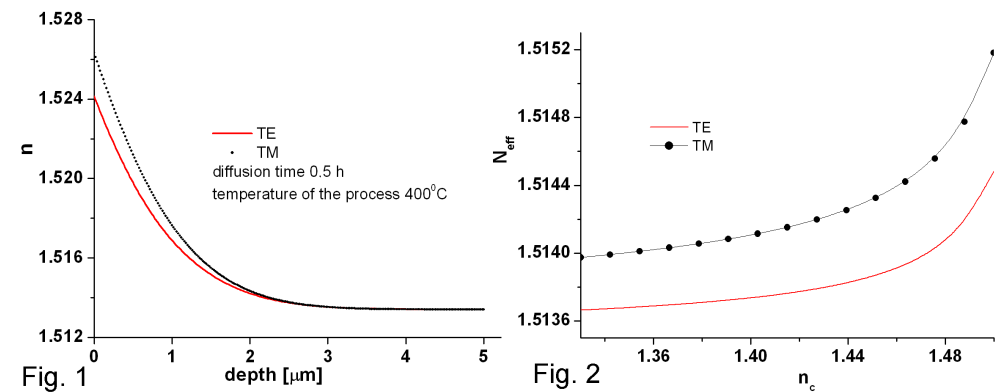

Fig. 1. Distribution of planar waveguide refractive indices made on glass BK7 in the ion exchange $\mathrm{K}^{+}-\mathrm{Na}^{+}$, at a diffusion time of $0.5 \mathrm{~h}$, temperature of the process $T=400^{\circ} \mathrm{C}$. The top curve corresponds to the polarization TM, the bottom to the polarization TE. Fig. 2. Influence of the refractive index of the waveguide cover $n_{\mathrm{c}}$ on the effective indices of the modes.

In many sensor applications we use the fact that the change of the refractive index of the cover $\mathrm{d} n_{\mathrm{c}}$ influences in various ways the effective indices of orthogonal modes, changing at the same time the phase difference $\mathrm{d}\left(\Delta \varphi_{0}\right)$ between the modes at the waveguide outlet. The mode phase $\varphi$ along the path of propagation may be expressed by the relation

$$
\varphi=\frac{2 \pi}{\lambda_{0}} N_{\mathrm{eff}} x
$$

where $x$ - working length of the interferometer, $N_{\text {eff }}$ - effective indices, $\lambda_{0}-$ wavelength. The dependence of the effective index of refraction at a wavelength of $\lambda_{0}=670 \mathrm{~nm}$ for the fundamental modes TE and TM on the refraction index can be seen in Fig. 2. The phase velocity of the fundamental mode TE is higher than the phase velocity of the fundamental mode TM when the value of the refracting index of the cover amounts to $1.33 \div 1.50$. 
In the system of a difference interferometer the phase difference between orthogonal modes, resulting from difference between the effective refracting index is of essential importance

$$
\Delta \varphi=\frac{2 \pi}{\lambda_{0}}\left(\Delta N_{\text {eff }}\right) x
$$

where $x$ - working length of the interferometer, $\Delta N_{\text {eff }}$ - difference between the effective indices, $\lambda_{0}$ - wavelength.

The dependence of the difference between the effective refracting index $N_{\mathrm{TM}}$ and $N_{\mathrm{TE}}$ on the refraction index was presented in Fig. 3. An experimental verification has proved the compatibility of the theoretical analysis with the performed measurements.

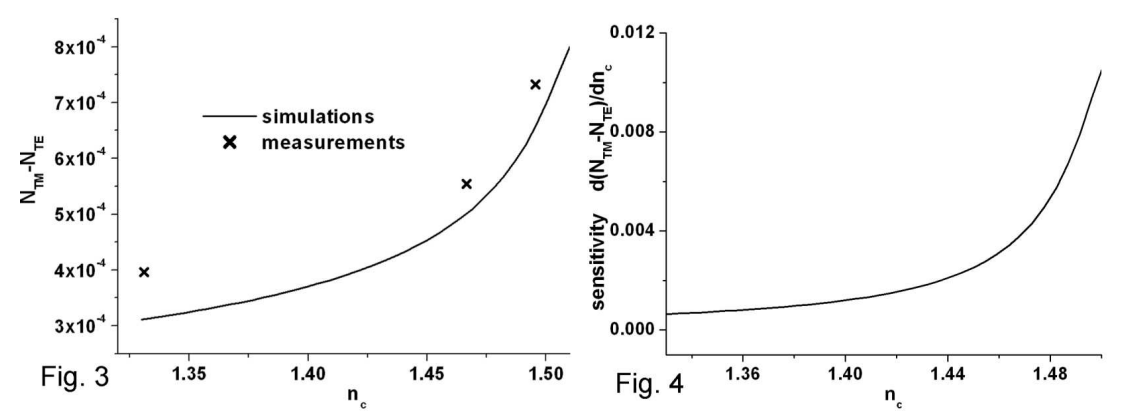

Fig. 3. Influence of the refractive index of the waveguide cover $n_{\mathrm{c}}$ on the difference between the effective indices of the modes.

Fig. 4. Influence of the refractive index of the waveguide cover $n_{\mathrm{c}}$ on the sensitivity.

An essential parameter for the differential interferometer is the sensitivity $S$, indicating in which degree a change of the refracting index of the cover $n_{\mathrm{c}}$ affects the difference between the effective refractive indices of the applied modes

$$
S=\left|\frac{\mathrm{d}\left(\Delta N_{\mathrm{eff}}\right)}{\mathrm{d} n_{\mathrm{c}}}\right| .
$$

The determined dependence of the sensitivity $S$ on the refractive index of the cover was presented in Fig. 4. The weakest sensibility occurs when the refracting index of the cover is of the range 1.33 about 0.0007 , changing slightly to the value $n_{\mathrm{c}}=1.44$. Above the value 1.44 the sensitivity increases to about 0.01 for $n_{\mathrm{c}}=1.5$.

\section{Composite waveguide structures}

Planar waveguides in which some range of the waveguide has been modified by depositing on it a layer of some other dielectric, are called composite optical waveguides. This kind of structure is of much interest due to considerable possibilities of taking measurements, which may contribute to a further development of the optoelectronic sensor. Especially required are sensor systems in chemistry, 
biochemistry, biotechnology, and others. Investigations on the properties of composite layers constitute a continuation of investigations concerning the construction of interferometers, which are very sensitive, cheap and easy to be produced [5-8].

Composite waveguides which are actually being investigated are structures of thin dielectric layers narrowed at their ends, deposited on definite surfaces of planar waveguides [5-10]. The reduced parts of the layer play the role of couplers, which have to introduce into the composite structure as much optical power as possible.

Such a structure is based on a planar waveguide obtained by means of ion exchange $\mathrm{K}^{+}-\mathrm{Na}^{+}$on glass, and covered by an adequately profiled dielectric layer.

The application of a waveguide obtained by ion exchange as the basic structure is recommendable due to several advantages:

- a low attenuation - the possibility of detecting the output signal even after a long path of propagation,

— low refracting index - the possibility of applying various materials with a higher refracting index as covering layers,

- low sensibility to polarization - the possibility of a simultaneous excitation of $\mathrm{TE}_{0}$ and $\mathrm{TM}_{0}$ modes in one beam.

The principle of operation of such a coupler consists in the gradual change of the wave vectors of the coupled mode with the distance. If the coupler is to transfer the most possible power from one waveguide area to the other, the coupling zone must be sufficiently long and the angle of inclination adequate. In the case of visible light the length of the coupler ought to amount to 100-1000 times of the wavelength. The diagram of a composite waveguide can be seen in Fig. 5 .

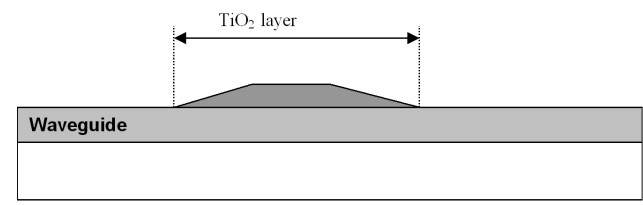

Fig. 5. Longitudinal cross-section of a composite waveguide structure.

The refracting index of a composite layer $\left(\mathrm{TiO}_{2}\right.$ layer $)$ is larger than the refracting index of the waveguide layer, thanks to which the evanescent field in the composite structure is larger than the evanescent field in a waveguide obtained by means of the ion exchange technique. The dimension of the evanescent fields is the crucial parameter, influencing the sensitivity of sensors basing on the absorption of the evanescent wave or interference. If at a given refracting index $n_{\mathrm{f}}$ of the additional layer the thickness of the layer exceeds the thickness which permits the propagation of the $\mathrm{TE}_{0}$ mode, being however smaller than the thickness permitting the propagation of the $\mathrm{TM}_{0}$ mode, then due to the resolution of polarization the 
mode $\mathrm{TE}_{0}$ penetrates from the waveguide obtained by means of the ion exchange technique into the additional layer.

The profile applied in calculations of the refraction index was presented in Fig. 6. To the profile of the refraction index of the waveguides obtained by ion exchange, a layer was added with a thickness of $40 \mathrm{~nm}$ and the refracting index 2.3. The dependence of the effective refracting index at the wavelength $\lambda_{0}=670 \mathrm{~nm}$ for concerning the fundamental modes TE and TM on the refracting index of the cover is to be seen in Fig. 7. The phase velocity of the fundamental mode TM is in this case larger than the phase velocity of the fundamental mode TE if the values of the refraction index of the cover are contained within the range from 1.33 to 1.50 .
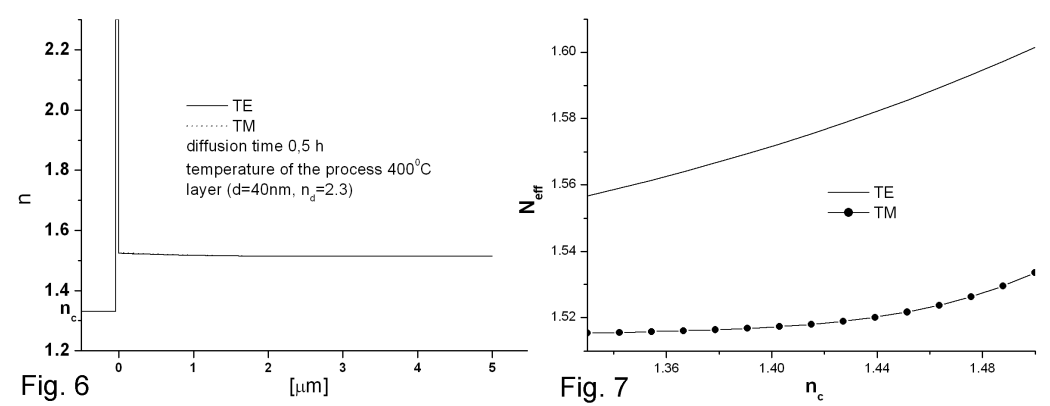

Fig. 6. Distribution of planar waveguide refractive indices for composite waveguide structures (ion exchange $\mathrm{K}^{+}-\mathrm{Na}^{+}$in glass BK7 for the diffusion time $0.5 \mathrm{~h}$, temperature of the process $T=400^{\circ} \mathrm{C}$, and the $\mathrm{TiO}_{2}$ composite layer).

Fig. 7. Influence of the refractive index of the waveguide cover $n_{\mathrm{c}}$ on the effective indices of the modes.
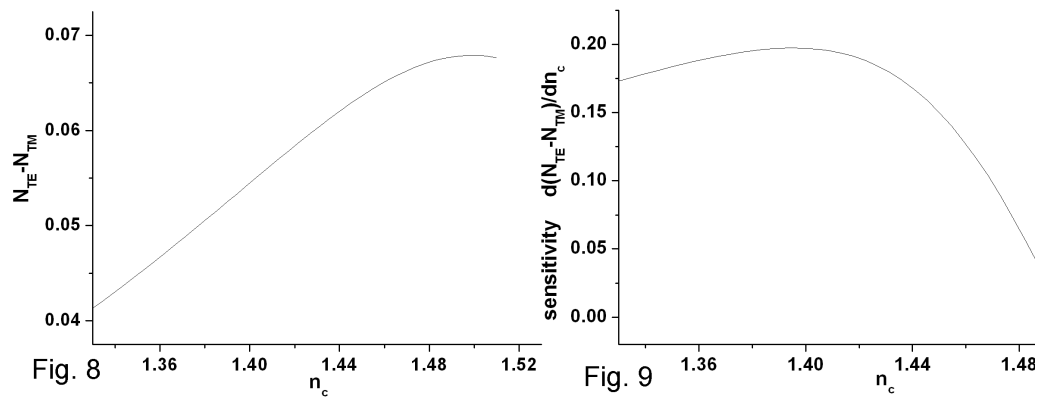

Fig. 8. Influence of the refractive index of the waveguide cover $n_{\mathrm{c}}$ on the difference between the effective indices of the modes.

Fig. 9. Influence of the refractive index of the waveguide cover $n_{\mathrm{c}}$ on the sensitivity.

The dependence of the difference between the effective refracting indices $N_{\mathrm{TE}}$ and $N_{\mathrm{TM}}$ on the refracting index of the cover was presented in Fig. 8 . The 
difference between the effective refracting indices increases almost linearly with the growing refracting index of the cover $n_{\mathrm{c}}$.

The dependence of the sensitivity $S_{\text {com }}$ of the refracting index of the cover, calculated in this case was presented in Fig. 9. The sensitivity $S_{\text {com }}$ of the composite waveguide is comparatively high in the range of changes of $n_{\mathrm{c}}$ from 1.33 to 1.44 , amounting to about 0.17 .

\section{Conclusions}

The paper presents the fundamental parameters of a planar difference interferometer, with a planar waveguide, obtained as a result of ion exchange on glass type BK7. The sensitivity of the interferometer was determined for two cases, the planar waveguide proper and a planar waveguide with a nanometric layer with a high refracting index. In the analysis of this system various profiles of the refracting index for the polarization of TE and TM were taken into account. In the range of changes of $n_{\mathrm{c}}$ from 1.33 to 1.38 the sensitivity of the interferometer with a nanometric layer is more than 200 times higher than in a system without such a layer.

System of this type i particularly applicable in biochemistry, where it monitors the changes of the refracting indices in the range from 1.33 to 1.4.

\section{Acknowledgments}

This work was supported by the Ministry of Science and Higher Education within the grant R01 03901.

\section{References}

[1] T. Pustelny, Physical and Technical Aspects of Optoelectronic Sensors, Publisher of Silesian University of Technology, Gliwice 2005.

[2] T. Pustelny, K. Barczak, K. Gut, J. Wojcik, Opt. Appl. 34, 531 (2004).

[3] C. Tyszkiewicz, T. Pustelny, J. Phys. IV (France) 129, 169 (2005).

[4] T. Pustelny, C. Tyszkiewicz, Opt. Appl. 34, 507 (2004).

[5] Z. Qi, A. Yimit, K. Itoh, M. Murabayashi, H. Yanagi, J. Lightwave Technol. 18, $1106(2000)$.

[6] Z. Qi, K. Itoh, M. Murabayashi, A. Yimit, H. Yanagi, J. Electrochem. Soc. 147, $3940(2000)$.

[7] K. Gut, Mol. Quant. Acoust. 26, 91 (2005).

[8] Z. Qi, A.Yimit, K. Itoh, M. Murabayashi, Opt. Lett. 26, 629 (2001).

[9] Z. Qi, A. Yimit, K. Itoh, M. Murabayashi, Opt. Lett. 25, 1427 (2000).

[10] B. Pustelny, T. Pustelny, Mol. Quant. Acoust. 28, 223 (2007).

[11] R. Rogoziński, K. Gut, P. Karasiński, A. Opilski, Proc. SPIE 3581, 375 (1998). 